DE

M E D I C I N A

T R O P I C A L

$\mathrm{DE}$

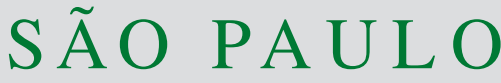

JOURNAL OF THE SÃO PAULO INSTITUTE OF TROPICAL MEDICINE

(1) Universidade de São Paulo, Faculdade de Medicina, Departamento de Moléstias Infecciosas e Parasitárias, São Paulo, São Paulo, Brazil

(2) Universidade de São Paulo, Instituto de Medicina Tropical de São Paulo, Laboratório de Parasitologia, São Paulo, São Paulo, Brazil

(3) Universidade de São Paulo, Faculdade de Medicina, Departamento de Patologia, Laboratório de Patologia de Moléstias Transmissíveis, São Paulo, São Paulo, Brazil

(4) Universidade de São Paulo, Instituto de Medicina Tropical de São Paulo, Laboratório de Protozoologia, São Paulo, São Paulo, Brazil

(5) Fundação Oswaldo Cruz, Instituto Nacional de Infectologia Evandro Chagas, Laboratório de Pesquisa Clínica e Vigilância em Leishmanioses, Manguinhos, Rio de Janeiro, Brazil

(6) Instituto Oswaldo Cruz (Fiocruz), Laboratório de Imunoparasitologia, Manguinhos, Rio de Janeiro, Brazil

Correspondence to: Valdir Sabbaga Amato.

Universidade de São Paulo, Instituto de Medicina Tropical de São Paulo, Av. Dr. Enéas de Carvalho Aguiar, 470, CEP 05403-000, São Paulo, SP, Brazil.

Tel: +55 11 3061-7010

E-mail: valdiramato@usp.br

Received: 10 October 2016

Accepted: 17 January 2017

\section{Reactivation of cutaneous and mucocutaneous tegumentary leishmaniasis in rheumatoid arthritis patients: an emerging problem?}

\author{
Regina Maia de Souza ${ }^{1,2}$, Heitor Franco de Andrade Junior ${ }^{3,4}$, Maria Irma \\ Seixas Duarte ${ }^{3}$, Lucia Maria Almeida Braz², Armando de Oliveira Schubach ${ }^{5}$, \\ Fátima Conceição Silva ${ }^{6}$, Valdir Sabbaga Amato, ${ }^{1,2}$
}

\section{ABSTRACT}

Rheumatoid arthritis (RA) is a chronic condition that is frequent in patients living in tropical areas exposed to leishmaniasis. RA therapy involves immunosuppressant drugs such as methotrexate (MTX), monoclonal antibodies (mAbs) and prednisone. We report an unusual presentation of cutaneous (CL) or mucocutaneous leishmaniasis (ML) in RA patients from an endemic area of leishmaniasis. A 51-year-old woman noted a cutaneous ulcer on her left ankle during MTX and prednisone RA therapy. Initially diagnosed as a venous stasis ulcer, the aspirate of the injury revealed the presence of Leishmania DNA. A 73-year-old woman presenting non-ulcerated, infiltrated and painful erythematous nodules inside her nostrils while receiving MTX, anti-TNF mAb, and prednisone for RA, had also the aspirate of injuries showing the presence of Leishmania DNA. Both patients healed after the therapy with liposomal amphotericin. The RA therapy has changed to low-dose prednisone, without further reactivation episodes. Both cases suggest that CL or ML can reactivate after administration of an immunosuppressant for RA treatment. Therefore, immunosuppressive treatments for RA should be carefully prescribed in patients from endemic areas or with a history of CL and ML.

KEYWORDS: Leishmaniasis. Reactivation. Methotrexate. Anti-TNF mabs. Rheumatoid arthritis.

\section{INTRODUCTION}

Leishmaniasis is an anthropozoonosis caused by Leishmania species and can have different clinical presentations depending on the parasite-host relationship ${ }^{1}$. The most common species of Leishmania in Brazil are L. (Viannia) braziliensis, L. (Leishmania) amazonensis, and L. (V.) guyanensis, followed by the uncommon species L. (V.) naiffi, L. (V.) lainsoni, L. (V.) shawi, and L. (V.) lindenbergi ${ }^{2}$. Leishmania (V.) braziliensis has been more frequently found in the South and Southeast regions of Brazil.

Cutaneous leishmaniasis (CL) and mucocutaneous leishmaniasis are widely distributed in Brazil, and although they are considered to be less severe than the visceral forms, they produce cutaneous (CL) and/or mucosal (ML) lesions capable of mutilating or stigmatizing the patients, making the social inclusion of affected individuals difficult.

The lesions appear primarily in uncovered areas of the skin, often as ulcers with well-defined edges and a granular bottom. In some individuals, the patient may also 
experience lesions of the mucosal tissue, especially of the upper respiratory and digestive tract, producing discomfort swallowing or breathing. Oftentimes, the infections are chronic and can reactivate several times during the patient's life, since parasites are known to persist at the lesions ${ }^{3,4}$.

Rheumatoid arthritis (RA) therapy is a rapidly evolving area of study involving the use of potent immunosuppressant drugs, such as methotrexate, and anticytokine immunobiologicals ${ }^{5}$. In addition to the classical prednisone therapy, these newer strategies have significantly modulated the host immune response, increasing the patient's susceptibility to infections, such as leishmaniasis ${ }^{6}$. Herein, we present two patients with reactivated cutaneous or mucocutaneous leishmaniasis after immunosuppressive therapies against RA, and discuss that the specific therapy for RA must consider the risk for CL or ML leishmaniasis reactivation.

\section{CASE PRESENTATION}

\section{Case report 1}

A 51-year-old Brazilian woman presented with a cutaneous ulcer on her left ankle and was treated in the Division of Infectious and Parasitic Diseases, Hospital das Clínicas, University of São Paulo, Medical School, São Paulo, Brazil. She reported had been receiving various drugs to control rheumatoid arthritis, diagnosed several years before. The treatment schedule included $25 \mathrm{mg}$ of methotrexate intramuscularly once a week for two years, $5 \mathrm{mg}$ of prednisone orally and daily for five years, and chloroquine $400 \mathrm{mg}$ orally and daily for two years. During her childhood, the patient used to live in an endemic county for cutaneous leishmaniasis (São Lourenço da Serra - São Paulo) and described the presence of a long-lived skin ulcer, which had appeared at that time and diagnosed as cutaneous leishmaniasis. At examination, the lesion presented as a painful, foul smelling, and edematous $8 \mathrm{~cm}$-diameter ulcer in the fibular malleolus region, which showed fibrin but without infiltrated edges (Figure 1A). Another atrophic circular scar has also been found on the left pretibial region. Histology of the biopsy obtained from the border of the lesion was compatible with a venous stasis ulcer with intense granular tissue and scarring (Figure 1B). The patient had started empirical treatment with piperacillin, tazobactam and clindamycin. The patient developed fever, but with no growth in blood cultures. The patient's treatment was altered to imipenem $500 \mathrm{mg} /$ day for 7 days resulting in improvement in the patient's condition and renal function. During the investigation, Leishmania PCR analysis from the aspirate of the lesion was positive (Figure 1C), resulting in treatment with $3 \mathrm{mg} / \mathrm{kg} /$ day of a liposomal amphotericin protocol for ten days (total cumulative dose of $35 \mathrm{mg}$ ). The possible reactivation of the cutaneous leishmaniasis diagnosed during her childhood was considered at this point. During the last few doses, amphotericin treatment was discontinued due to worsening of the renal function, but the treatment was completed after dose adjustments for $2 \mathrm{mg} / \mathrm{kg} /$ day. In addition, to control RA, $20 \mathrm{mg} / \mathrm{day}$ of leflunomide was introduced after the rheumatologist evaluation. After the treatment with amphotericin was finished, the cutaneous ulcer on the left ankle of the patient had clearly improved without fibrin, as noted through visual inspection. At this time, the patient was discharged from hospital and continued to be monitored at her hometown until the ulcer had completely healed (Figure 1D).

\section{Case report 2}

A 73-year-old Brazilian woman was admitted to the medical service presenting a lesion on her face (Figure 2A). She had a history of leishmaniasis at the age of nine years-old. Four years ago, she presented with painful erythematous nodules on her face, more precisely in the nasal passages, nose, and perioral region. The lesions were diagnosed as mucocutaneous leishmaniasis and treated with $20 \mathrm{mg} / \mathrm{kg} / \mathrm{day}$ of pentavalent antimony (Glucantime ${ }^{\circledR}$ ) for twenty-eight days, resulting in their disappearance. After a nasofibroscopy, it was determined that the patient was clinically cured. However, six months ago, she began to suffer from pain in her face and the reappearance of the infiltrated erythematous nodules, which were very similar to those that had appeared 4 years before. The patient was again hospitalized, and during anamnesis, she reported that she had received a weekly treatment for rheumatoid arthritis with $7.5 \mathrm{mg}$ of methotrexate, $6 \mathrm{mg}$ of prednisone, $100 \mathrm{mg}$ of chloroquine, and $50 \mathrm{mg} / \mathrm{month}$ of golimumab. At this time, the small erythematous lesions had become an infiltrated plate in the septal region associated with ulcerated areas with intranasal crusts. The patient began an empirical treatment for rhinitis with nasal topical medication. The lesion progressively evolved, and the Montenegro intradermal test showed a $15-\mathrm{mm}$ diameter induration. A diameter of $\geq 5 \mathrm{~mm}$ indicates a positive reaction. The Montenegro intradermal test is useful in cases in which the parasites are scarce or absent in the lesions, i.e., when acute cutaneous leishmaniasis lesion has undergone more than 2 months of evolution and spontaneous healed ${ }^{7}$. Histological evaluation (HE) of the biopsy border showed Leishmania amastigotes and an inflammatory infiltrate (Figure 2B). kDNA-PCR analysis of the lesion aspirate showed the presence of Leishmania sp. The amplification product of the 


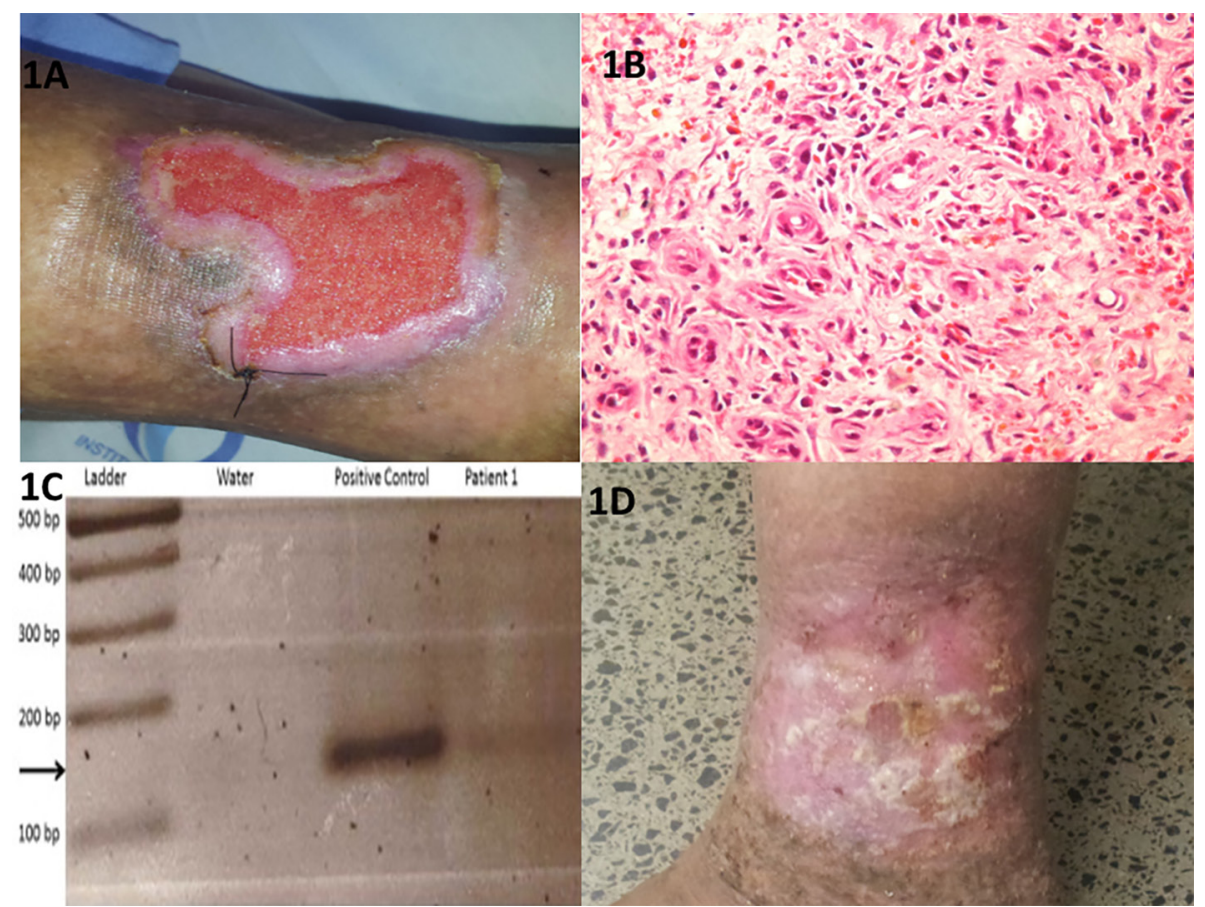

Figure 1 - Lesion on the left ankle of patient 1 before therapy at biopsy (1A); histology of the initial biopsy (1B) showed granulated tissue and signs of venous stasis (HE x400); kDNA-PCR analysis amplified a 120-bp fragment (1C); the lesion after completing anti-leishmanial and antimicrobial therapy is shown (1D).

kDNA-PCR was subjected to Restriction Fragment Length Polymorphism (RFLP) and the profile was compatible with L. (V.) braziliensis ((fragments of 40 and $80 \mathrm{bp})^{8}$, as seen in the Figure $2 \mathrm{C}$, confirming the diagnosis of ML. Thirty-five $\mathrm{mg} / \mathrm{kg}$ of liposomal amphotericin B was used for treatment. After the fourth infusion, laboratory tests revealed that the patient had nephrotoxicity manifested with polyuria, leading to a daily correction of doses, ultimately resulting

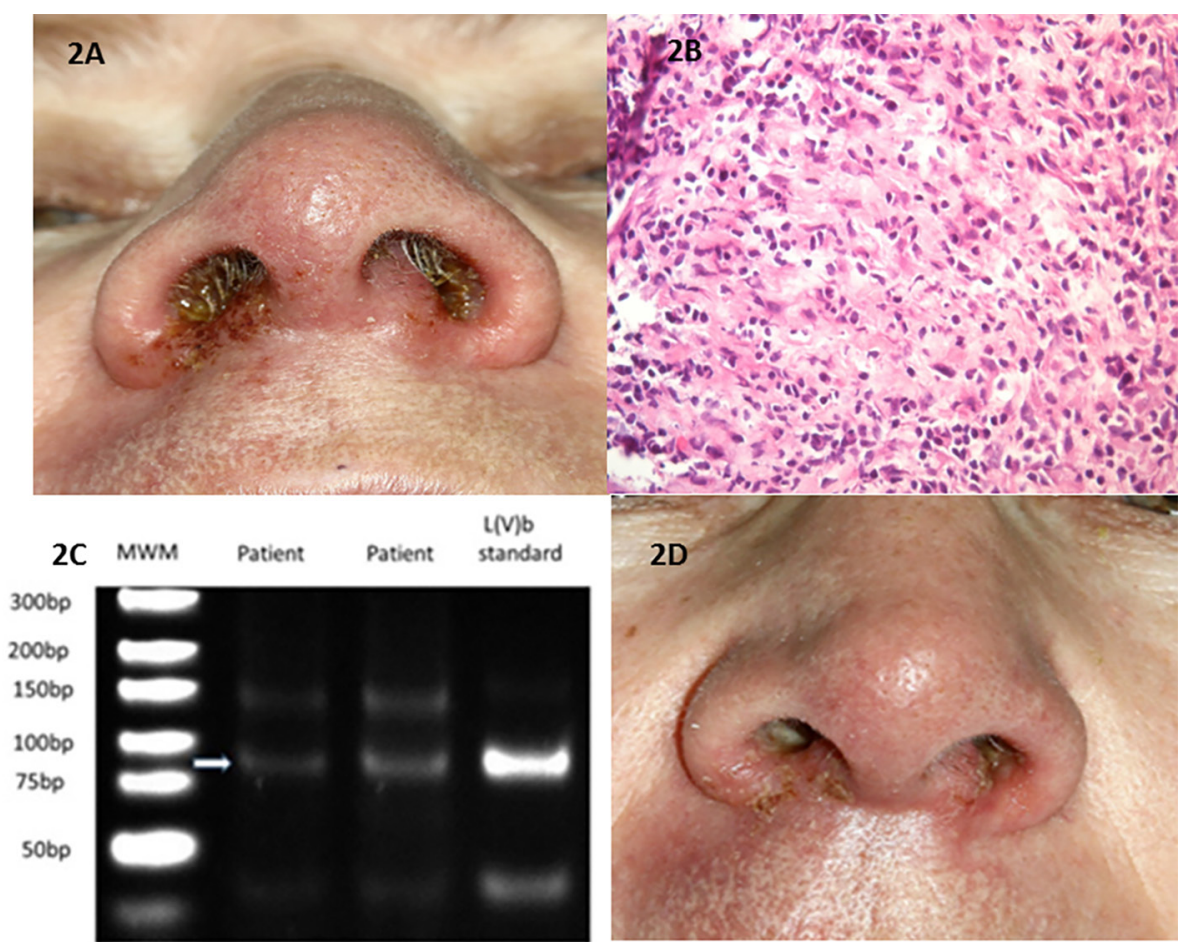

Figure 2 - Induration and crusting on the nasal areas of the lesion of patient 2 at admission (2A); results of histology (2B) (HE $\mathrm{x} 400$ ); PCR-RFLP (2C) showing the 80 and the 40-bp fragments, and the lesion after completion of Leishmania-specific therapy is shown (2D). 
in improved renal function. The rheumatology group advisor suggested that treatment of rheumatoid arthritis could be maintained only with $10 \mathrm{mg} / \mathrm{day}$ of prednisone administered twice a day $(5 \mathrm{mg} /$ dose $)$ to prevent adrenal insufficiency. After receiving a cumulative dose of 2,500 $\mathrm{mg}$ of amphotericin B, the patient showed a significant clinical improvement (Figure 2D), when compared to Figure 2A. At this time, she was discharged from hospital and was followed at the outpatient clinic for treatment and surveillance of her leishmaniasis, perioral lesions and RA.

\section{DISCUSSION}

We described two confirmed cases of reactivated CL or ML leishmaniasis in two patients who lived in endemic areas, had a history of previous contact with the Leishmania parasite and were under immunosuppression due to therapy for rheumatoid arthritis. The first case appears to be a reactivation of a single cutaneous leishmaniasis lesion due to parasites that remained in the old scar, after immunosuppression. In case 2, there was reactivation of mucosal leishmaniasis, which has been associated with hyperactive immune responses in immunocompetent patients. However, mucosal lesions can also be found in HIV-AIDS co-infected patients ${ }^{9}$, suggesting that the deregulation of the specific immune response, as produced by $\mathrm{mAb}$ therapy, can play a role in the lesion development. The lesions of both patients resolved with anti-leishmania therapy, and the RA treatments were adjusted to decrease the level of immunosuppression.

The immune response can either favor, cure, or lead to disease development, depending on its quality and intensity. When immunosuppression is present, this instability affects the patient's immune control of pathogens or cancer cells. Consequently, the development of severe infections or neoplasia can take place. On the other hand, when hyperactivity is present, allergies, autoimmune diseases, and uncontrolled tissue damage become common.

In this context, what can occur when both branches are present? Cutaneous and mucosal leishmaniasis stimulate a predominant type 1 immune response, characterized by a high-level of IFN- $\gamma$ and TNF- $\alpha$ secretion ${ }^{10}$. Th1 immune responses, although associated with the control of parasite multiplication and dissemination, can cause tissue damage if they are not modulated ${ }^{11,12}$. The protozoa can persist alive in the scars of ancient lesions for years, sustained by a persistent, well-modulated Th1 local immune response pattern, even after treatment ${ }^{4}$. A role for TNF- $\alpha$ in the pathogenesis of mucosal leishmaniasis and other chronic inflammatory parasitic diseases is suggested by the increased levels of TNF- $\alpha$ in sera ${ }^{11}$, the high expression levels in mucosal tissue ${ }^{13}$, and the decreased TNF- $\alpha$ levels after therapy of mucosal leishmaniasis ${ }^{14}$. The literature suggests that the reactivation of leishmaniasis is associated with the deregulation of the specific immune response as a decrease in inflammatory cytokines (IFN- $\gamma$ and TNF- $\alpha$ ) and an increase of cytokines associated with the Th2 immune response (IL-4 and IL-10) ${ }^{15}$. An early prospective study published in 1994 suggested that patients receiving methotrexate had a higher risk of serious infection than those not receiving the drug, RR 1.52 (95\% CI $1.04-2.13)^{16}$. Data from the US CORRONA study group (http://www. corrona.org) found a similarly increased risk of infection and an incidence rate ratio of 1.30 (95\% CI 1.12, 1.50) in a prospective follow-up of a cohort of 7,971 RA patients when comparing MTX with other disease modifying antirheumatic drugs (DEMARDs). There is also a reported case of tuberculous pericarditis complicated by cardiac tamponade in an elderly patient on methotrexate treatment for rheumatoid arthritis ${ }^{17}$. Anti-TNF- $\alpha$ therapy has also been associated with an increased risk for developing opportunistic infections ${ }^{18}$ such as Pneumocystis jirovecii pneumonia, histoplasmosis, cytomegalovirus infections, aspergillosis, cryptococcal meningitis, leishmaniasis, and, in particular, tuberculosis ${ }^{19}$.

Despite an increase amount of information, most physicians still have problems associating the presence of cutaneous and mucosal lesions with infection or reactivation of $\mathrm{CL}$ or ML leishmaniasis in patients under immunosuppressive treatment. These patients may have some epidemiological evidence of previous leishmaniasis, but are, at the time of diagnosis, living in non-endemic regions of neglected tropical diseases. In this context, it is still necessary to emphasize the possibility of the immunossupressive treatment be responsible for reactivating leishmaniasis, primarily in older people living in non-endemic areas/ countries but also in patients with a history of living or traveling to CL and ML-endemic areas. Reports from a non-endemic country, such as the USA, have shown that the infection rates are the highest among previously exposed immigrants from endemic areas ${ }^{20,21}$.

\section{CONCLUSIONS}

Immunosuppressive treatments for RA could be associated with reactivation of cutaneous or mucocutaneous leishmaniasis. Our reported cases must alert physicians from non-endemic regions/ countries to the possibility of leishmaniasis whenever unusual infections occur in patients starting immunosuppressant RA treatment, especially when they have previously lived abroad, and in older, migrant 
patients. This problem calls for increased information, surveillance, and the availability of diagnosis and treatment of cases, which is difficult in non-endemic countries. Information from RA immunosuppressive treatment with disease-modifying anti-rheumatic drugs (DMARDs) and biologicals, such as mAbs, must alert physicians to the risk of reactivation of latent $C L$ and ML leishmaniasis in patients who lived or traveled to leishmaniasis-endemic areas.

\section{ETHICS APPROVAL AND CONSENT TO PARTICIPATE}

This study was approved for submission to scientific journals, Number 016/15, by the Committee of Ethics for Analysis of Research Projects (CAPPesq - Comitê de Ética para Análise de Projetos de Pesquisa - Hospital das Clínicas da Faculdade de Medicina da Universidade de São Paulo). The patients records/information were anonymized and did not identify them. The pictures used were authorized by the patients, and authorizations were recorded in the patient's charts.

\section{COMPETING INTERESTS}

The authors declare that they have no competing interests.

\section{AUTHORS' CONTRIBUTIONS}

RMS, HFAJ, MISD, LMAB and VSA have participated in the planning of the study. RMS has the responsibility for the molecular diagnosis. MISD was responsible for histopathological analyses and VSA helped to treat the patients. RMS, HFAJ, MISD, LMAB, AOS, FCS and VSA were involved in the discussion of results and the drafting/ revision of the manuscript. They have read and approved the final manuscript.

\section{ACKNOWLEDGEMENTS}

We gratefully thank all of the personnel involved in the primary care of our patients. This project was supported by FAPESP 2010/503048 and LIMHCFMUSP 46 and 49. HFAJ is a fellow of CNPq and FFM.

\section{REFERENCES}

1. Brasil. Ministério da Saúde. Secretaria de Vigilância em Saúde. Manual de vigilância da leishmaniose tegumentar americana. Brasília: Ministério da Saúde; 2007. p.13.

2. Bacha HA, Tuon FF, Zampieri RA, Floeter-Winter LM, Oliveira
J, Nicodemo AC, et al. Leishmania (Viannia) braziliensis identification by PCR in the state of Para, Brazil. Trans R Soc Trop Med Hyg. 2011;105:173-8.

3. Morgado FN, Schubach A, Vasconcellos E, Azeredo-Coutinho RB, Valete-Rosalino CM, Quintella LP, et al. Signs of an in situ inflammatory reaction in scars of human American tegumentary leishmaniasis. Parasite Immunol. 2010;32:285-95.

4. Schubach A, Marzochi MC, Cuzzi-Maya T, Oliveira AV, Araujo ML, Oliveira AL, et al. Cutaneous scars in American tegumentary leishmaniasis patients: a site of Leishmania (Viannia) braziliensis persistence and viability eleven years after antimonial therapy and clinical cure. Am J Trop Med Hyg. 1998;58:824-7.

5. Brenol CV, da Chakr RM, Andrade NP, Toni M, Laurindo IM, Brenol JC, et al. Daily practice feasibility and effectiveness of treating long-standing rheumatoid arthritis to target with synthetic disease-modifying antirheumatic drugs: a prospective cohort study. Clin Rheumatol. 2015;34:1781-5.

6. Mueller MC, Fleischmann E, Grunke M, Schewe S, Bogner JR, Löscher T. Relapsing cutaneous leishmaniasis in a patient with ankylosing spondylitis treated with infliximab. Am J Trop Med Hyg. 2009;81:52-4.

7. Skraba CM, de Mello TF, Pedroso RB, Ferreira ÉC, Demarchi IG, Aristides SM, et al. Evaluation of the reference value for the Montenegro skin test. Rev Soc Bras Med Trop. 2015;48:437-44.

8. Volpini AC, Passos VM, Oliveira GC, Romanha AJ. PCR-RFLP to identify Leishmania (Viannia) braziliensis and L. (Leishmania) amazonensis causing American cutaneous leishmaniasis. Acta Trop. 2004;90:31-7.

9. Amato VS, Tuon FF, Camargo RA, Souza RM, Santos CR, Nicodemo AC. Can use a lower dose of liposomal amphotericin $\mathrm{B}$ for the treatment of mucosal American leishmaniasis? Am J Trop Med Hyg. 2011;85:818-9.

10. Nylén S, Eidsmo L. Tissue damage and immunity in cutaneous leishmaniasis. Parasite Immunol. 2012;34:551-61.

11. Bacellar O, Lessa H, Schriefer A, Machado P, Ribeiro de Jesus A, Dutra WO, et al. Up-regulation of Th1-type responses in mucosal leishmaniasis patients. Infect Immun. 2002;70:673440

12. Conceição-Silva F, Morgado FN, Costa-Santos M, Schubach AO, Oliveira-Mendes S. Leishmania braziliensis and in situ immune response: dispute or partnership? Rev Soc Bras Med Trop. 2010;43 Supl 2:64-71.

13. Amato VS, Andrade HF, Amato Neto V, Duarte MI. Short report: persistence of tumor necrosis factor-alpha in situ after lesion healing in mucosal leishmaniasis. Am J Trop Med Hyg. 2003; 68:527-8

14. Lessa HA, Machado P, Lima F, Cruz AA, Bacellar O, Guerreiro J, et al. Successful treatment of refractory mucosal leishmaniasis with pentoxifylline plus antimony. Am J Trop Med Hyg. 2001;65:87-9. 
15. Tuon FF, Bombonatto GM, Battaglin ER, Sakumoto MH, Amato VS, de Camargo RA, et al. Reactivation of mucosal and cutaneous leishmaniasis in a renal transplanted patient. Am J Trop Med Hyg. 2014;91:81-3.

16. van der Veen MJ, van der Heide A, Kruize AA, Bijlsma JW. Infection rate and use of antibiotics in patients with rheumatoid arthritis treated with methotrexate. Ann Rheum Dis. 1994;53:224-8.

17. Downey C. Serious infection during etanercept, infliximab and adalimumab therapy for rheumatoid arthritis: a literature review. Int J Rheum Dis. 2016;19:536-50.

18. Calabrò A, Caterino AL, Elefante E, Valentini V, Vitale A, Talarico $\mathrm{R}$, et al. One year in review 2016: novelties in the treatment of rheumatoid arthritis. Clin Exp Rheumatol. 2016;34:357-72.
19. Gomes KW, Benevides AN, Vieira FJ, Burlamaqui MP, Vieira MA, Fontenelle LM. Cutaneous leishmaniasis in a patient with ankylosing spondylitis using adalimumab. Rev Bras Reumatol. 2012;52:447- 52.

20. Amato VS, Tuon FF, Siqueira AM, Nicodemo AC, Neto VA. Treatment of mucosal leishmaniasis in Latin America: systematic review. Am J Trop Med Hyg. 2007;77:266-74.

21. Català A, Roé E, Dalmau J, Pomar V, Muñoz C, Yelamos O, et al. Anti-tumour necrosis factor-induced visceral and cutaneous leishmaniasis: case report and review of the literature. Dermatology. 2015;230:204-7. 